\title{
Application of Stochastic Regression for the Configuration of Microrotary Swaging Processes
}

\author{
Daniel Rippel, ${ }^{1}$ Eric Moumi, ${ }^{2}$ Michael Lütjen, ${ }^{1}$ Bernd Scholz-Reiter, ${ }^{1}$ and Bernd Kuhfuß ${ }^{2}$ \\ ${ }^{1}$ BIBA - Bremer Institut für Produktion und Logistik GmbH, University of Bremen, Hochschulring 20, 28359 Bremen, Germany \\ ${ }^{2}$ Bremen Institute for Mechanical Engineering, University of Bremen, Badgasteiner Straße 1, 28359 Bremen, Germany \\ Correspondence should be addressed to Daniel Rippel; rip@biba.uni-bremen.de
}

Received 31 January 2014; Accepted 5 March 2014; Published 1 June 2014

Academic Editor: Hamid Reza Karimi

Copyright (c) 2014 Daniel Rippel et al. This is an open access article distributed under the Creative Commons Attribution License, which permits unrestricted use, distribution, and reproduction in any medium, provided the original work is properly cited.

\begin{abstract}
In micromanufacturing, a precise adjustment of manufacturing, handling, and quality control processes constitutes an essential factor for success. The continuing miniaturization of workpieces and production devices results in ever decreasing tolerances, whereas machines and processes become increasingly more specialized. Thereby, the so-called size effects render the direct application of knowledge from the area of macromanufacturing impossible. In this context, this paper describes the application of the $\mu$-ProPlAn method for the configuration of an infeed rotary swaging process for microcomponents. At this, the cause-effect relationships between relevant process parameters are analyzed using stochastic regression models, in order to determine costefficient process configurations for the manufacturing of bulk and tubular microcomponents.
\end{abstract}

\section{Introduction}

During the last years, the demand of microcomponents increased strongly. Thereby, single components became increasingly smaller, while providing more functionality and consisting of more complex geometries [1]. In addition, increasing production rates and high demands regarding the components quality render the manufacturing of microcomponents a complex task. In this context, several authors define a microcomponent as being smaller than one millimeter in at least two geometrical dimensions [2]. One approach to achieving high throughput rates at comparably low costs is the application of cold forming techniques for micromanufacturing [3]. Different cold forming processes can be combined to achieve highly flexible manufacturing facilities with comparably low spatial requirements, for example, by applying desktop factories [4]. Such production systems face the challenge of producing vast amounts of high quality components while remaining cost efficient. Thereby, micromanufacturing is characterized by very low tolerances, the occurrence of so-called size effects [5], and a high degree of specialized manufacturing technologies.
As a result, the adjustment of a diversity of processes in micromanufacturing poses several challenges to the process designer. On the one hand, a careful selection of available process technologies is required. The suitability of certain process technologies strongly depends on the manufacturing context (e.g., materials, tools, preceding, or succeeding processes). Due to the high specialization, it might even be possible that processes have to be adapted or developed for a certain task. On the other hand, the adjustment of selected processes in a process chain has to be performed carefully. This includes manufacturing, handling, and quality control processes. Thereby, the design of process chains in micromanufacturing involves the configuration of relevant process parameters [6]. Such parameters cover material properties, machine settings, or specific tool configurations. Due to the low tolerances, slight changes to a single parameter can affect the overall process chain, causing high costs for setups or reconfigurations. Consequently, designers require additional support in the selection and adjustment of manufacturing, handling, and quality inspection processes in the area of micromanufacturing [7]. 


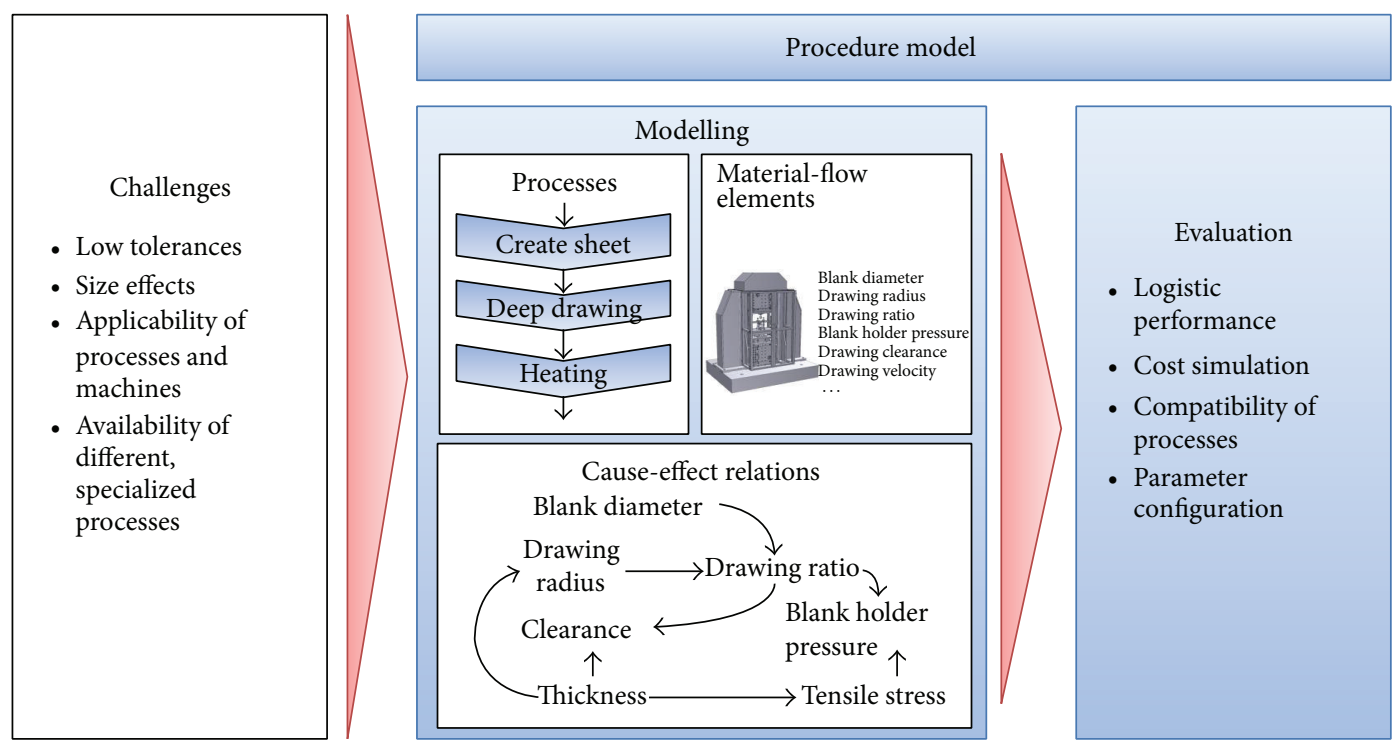

Figure 1: Components of the $\mu$-ProPlAn framework (after [11]).

In order to support process designers with the design and configuration of process chains in micromanufacturing, the $\mu$-ProPlAn (microprocess planning and analysis) framework (Figure 1) is being developed. The framework comprises methods to model and evaluate process chains in the context of micromanufacturing, covering all aspects from the process flow over material-flows to the modeling of socalled cause effect networks (Figure 1). These cause-effect networks describe correlations between technical and/or logistic parameters, within the microprocess chain. Moreover, the $\mu$ ProPlAn framework provides different methods to assess the quality of the designed process chains in terms of logistic performance and technical feasibility by computer-aided evaluations.

This paper demonstrates the application of $\mu$-ProPlAn for the configuration of a rotary swaging process in a micromanufacturing scenario. In contrast to classical process design methods, $\mu$-ProPlAn enables an integrated design and evaluation of the (logistic) process flow with respect to the manufacturing processes configurations. Thereby, statistical methods are applied to facilitate material-flow simulations by approximating the single manufacturing processes behaviors and results, based on experimental- or production-related data. The focus of this paper's case study is set on the production of high quality components, while avoiding costly setup operations by reconfiguring related process parameters. The next section introduces the area of micro-cold forming and describes particular challenges encountered in this area. Section 3 outlines the $\mu$-ProPlAn framework and its components. Afterwards, Section 4 describes the use-case scenario and the corresponding process models. Afterwards, it provides the simulation study conducted upon the use-case scenario and presents the results. The paper closes with a conclusion and outlines further work in this area.

\section{Micro-Cold Forming}

Cold forming processes provide a suitable approach to manufacturing high quality microcomponents, while maintaining high manufacturing accuracies and high throughputs. Additionally, workpieces usually become hardened during the cold forming process, resulting in products that are more robust. Compared to other manufacturing approaches, cold forming processes lead to a reduction of waste materials and energy consumption, leading to an environmentally friendly production [12].

Although cold forming processes are well known and widely used in macromanufacturing for mass production, they cannot be applied directly to micromanufacturing. The downscaling of those cold forming processes, and thus of the workpieces, tools, and machines, is only possible up to a certain degree. Thereafter, the impact of so-called size effects impedes a further downscaling of the cold forming process.

Vollertsen et al. define size effects as "deviations from intensive or proportional extrapolated extensive values of a process, which occur when scaling the geometrical dimensions" [2]. In this context, they define intensive values as parameters, which are not expected to change due to a change of an object's mass (e.g., its temperature or its density). In contrast, extensive values are expected to vary with a different mass (e.g., the object's inertia force or its heat content). Generally, size effects occur due to the inability to scale all relevant process parameters equally [2]. As an example, the downscaling of a metal sheet's thickness can result in a changing density due to local defects, although the density is considered an intensive variable. In addition to these effects, technical limitations further facilitate the occurrence of size effects. For example, the downscaling of mechanical grippers is limited by technical factors. For very small workpieces, the gripper's Van-der-Waals forces will eventually overcome 

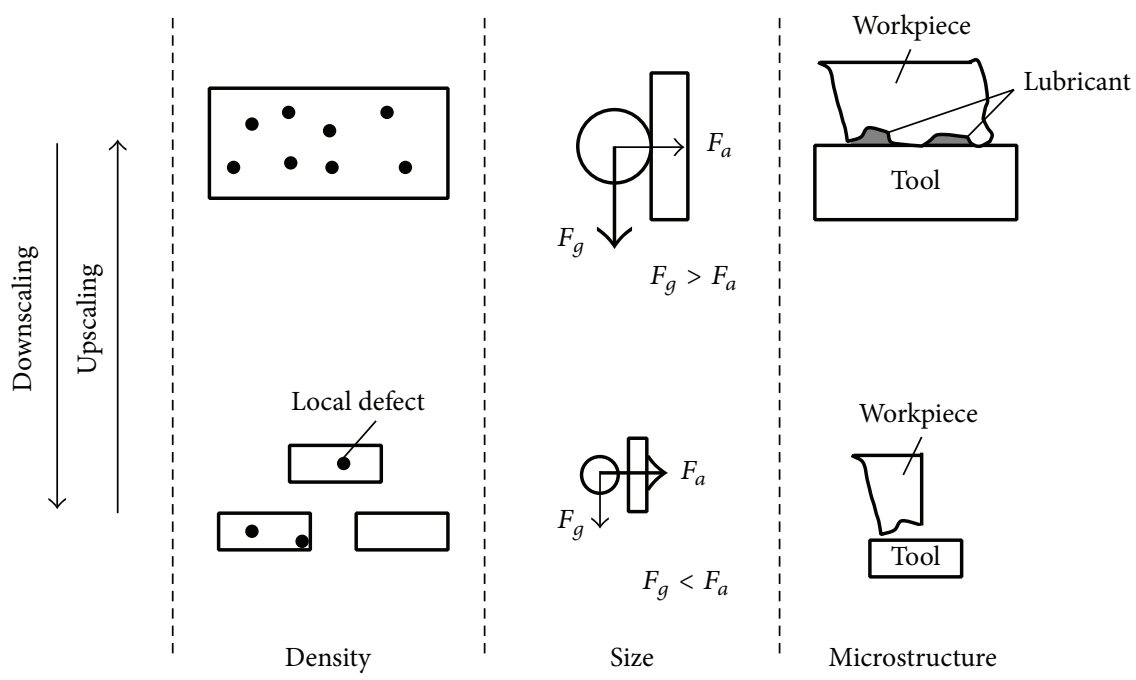

FIGURE 2: Categories of size effects [2].

the gravitational force at a certain point. Consequently, the gripper will not be able to release the workpiece without aid. Basically, Vollertsen and Walther define three distinct categories of size effects (Figure 2) [2].

(i) Density size effects occur, when the density of a material is held constant while scaling down its geometrical dimensions. For instance, local defects become more serious with a continuing miniaturization. Thereby, the distribution of local defects within a material can lead to more delimited sets of good and bad parts.

(ii) Shape size effects occur due to the increasing ratio of an object's total surface area, compared to its volume. An example of this category is provided by the described imbalance of the adhesive force in relation to the gravitational force.

(iii) Microstructure size effects occur because microstructural features (e.g., the grain size or the surface roughness) cannot be scaled down the same way as the geometrical size of an object.

The occurrence of size effects requires a precise planning of technical parameters throughout the overall process chain. Technical parameters between, as well as within, each manufacturing, handling, and quality-inspection process have to be regarded and adjusted to each other. Moreover, as new processes and technologies for micromanufacturing emerge quickly, interdependencies between those parameters cannot be described precisely or are unknown in several cases. As a result, the $\mu$-ProPlAn framework incorporates different methods to determine correlations between production relevant parameters by the application of stochastic methods from the fields of mathematics and artificial intelligence. These methods, on the one hand, enable a detailed analysis of cause-effect relations, while, on the other hand, they provide a suitable approach to determine parameter configurations based on production or experimental data.

\section{Microprocess Planning and Analysis ( $\mu$-ProPIAn)}

As depicted in Figure 1, $\mu$-ProPlAn consists of three basic parts. First, the modelling notation enables a clear and detailed modelling of the production system, covering manufacturing, handling, and quality inspection aspects throughout the process level, the material-flow level down to the configuration level. As a second part, a simultaneous engineering procedure model guides the modelling process. This procedure model aims at iteratively creating and adapting the model in parallel to the product development, enabling the detection of problems or inconsistencies early in the development phase. The third part finally covers the evaluation of the models. This includes the analysis of cause-effect relations during model creation, as well as the evaluation of the modelled production system concerning its technical feasibility and its logistic performance.

3.1. Modelling Notation. The modelling notation relies on the notation of process chains for the description of the overall process (Figure 3) $[6,13]$. Thereby, a process chain consists of a sequence of manufacturing, handling, and quality inspection processes. Each process again consists of one or more operations, relating to manufacturing, handling, or quality inspection tasks. The distinction between processes and operations enables the integration of several tasks in one process. For example, in-process quality inspections or simple transportation tasks can be represented as operations within a single process, if all of these tasks are conducted by a single workstation. In contrast, more complex tasks, for example, the separation of workpieces, can be represented as independent processes. In particular, complex quality inspection tasks often require several handling and inspection operations as a part of the overall quality inspection process to be conducted.

In order to adjust processes and operations to each other, the concept of process chains applies the so-called 


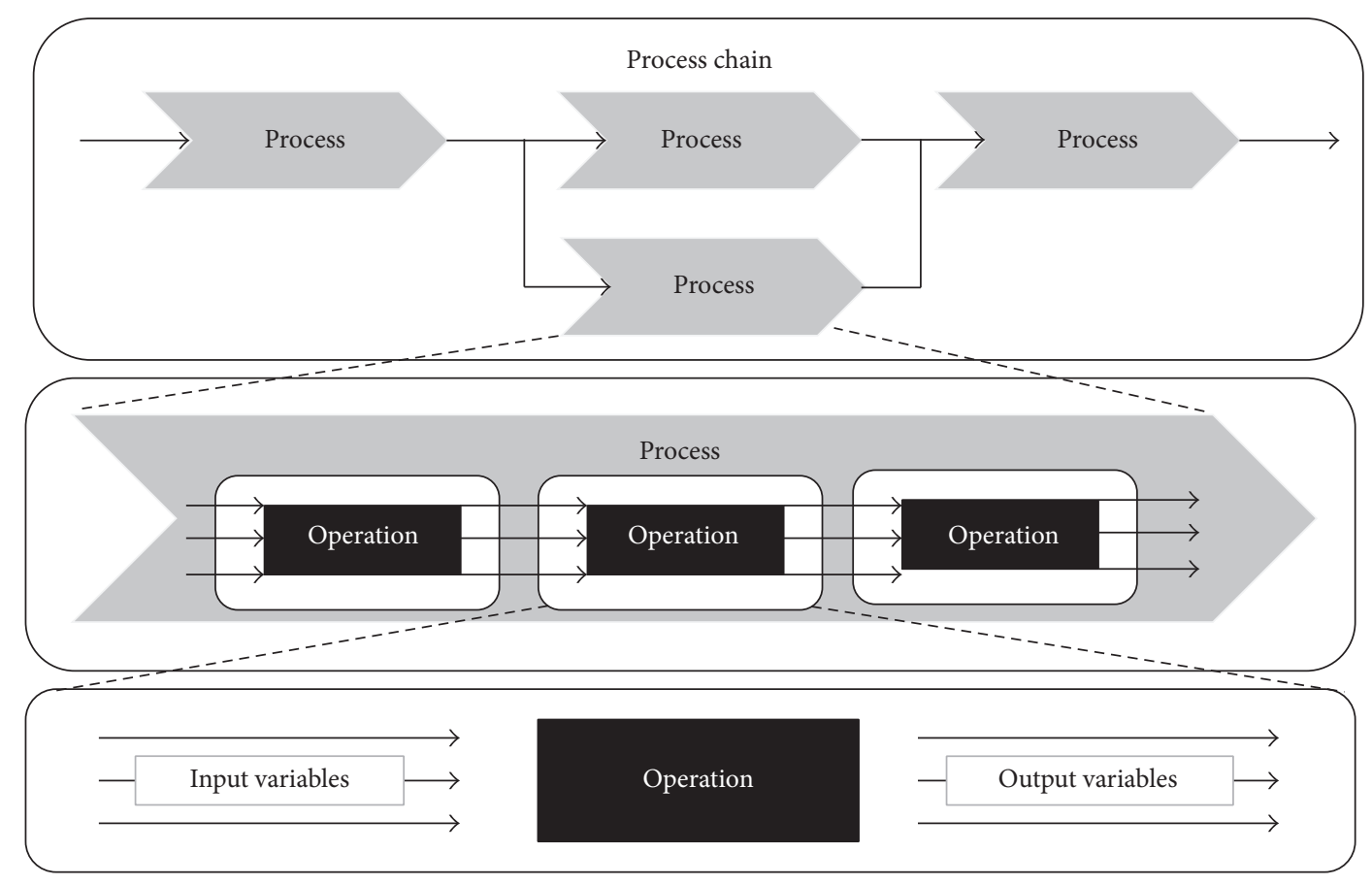

FIgURE 3: Structure of process chains (after [13]).

technological interfaces [13]. Therefore, each operation and, respectively, each process are assigned a set of technological input and output variables, which are modified during the operation. These variables usually constitute attributes of the workpiece, for example, geometrical features, its hardness, or surface roughness. Thereby, input variables describe the required state of the workpiece before the operation, while output variables describe the operations result.

While technological interfaces provide a powerful tool for the configuration of well-known processes in macromanufacturing, they are often inadequate for the configuration of processes in the microdomain. In micromanufacturing, the relationship between process parameters, related to machine or workstation settings, and the effects they have on the workpieces' properties are often unknown or underspecified. In addition, due to the very small tolerances inherent to the microdomain, even small changes to a process parameter can have a strong influence on the resulting workpiece's properties. Consequently, $\mu$-ProPlAn extends the classical concept of process chains by the so-called cause-effect networks. These networks consist of all relevant process and workpiece parameters and depict the cause-effect relations between each parameter. In order to minimize the modelling effort and to facilitate the reuse of cause-effect networks, they are modelled as a part of the respective material-flow element, for example, the machine, workpiece, or tool, and later on combined to cause-effect networks for an operation by assigning the material-flow elements used in the respective operation. Thus, each operation at least consists of an input and an output workpiece, usually constituting the operation's technological interfaces, as well as a machine and a tool, used to conduct the operation. Additionally, personnel and operating supplies can be assigned in order to enable a comprehensive logistic evaluation. Figure 4 shows a simplified cause-effect network for the operation "Rotary Swaging," at which several technological and logistic parameters were left out to increase the readability. This network is constructed from the causeeffect networks modelled for the rotary swaging machine (left), the respective workpiece (center), and the tool (right) used in this operation. Therefore, the single networks are connected via cause-effect relations between their parameters. In addition to the technological parameters, each causeeffect network contains a set of default logistic parameters. For example a tool requires the definition of its durability and its investment costs; operations require the specification of their duration and rejection rates, while machines provide parameters related to maintenance, malfunctions, and costs inflicted while processing or while being idle (for a complete list see [11]).

In order to enable the configuration of each operation and to enable a comprehensive technological and logistic evaluation of the modelled production system, the value of each parameter should be estimated based on its input parameters. Therefore, $\mu$-ProPlAn requires each parameter's cause-effect relations to be described quantitatively. In cases, in which the relationships are known, a mathematical function $y=$ $f\left(x_{1}, x_{2}, \ldots, x_{n}\right)$ can be defined for each parameter. In case of unknown cause-effect relations between the predictors and the dependent parameter, $\mu$-ProPlAn offers a set of methods to create an analytical regression model or to learn abstract prediction models, based on experimental or productionrelated data. A summary of these methods is provided in Table 1, together with a short description of each method. In addition to this quantitative description, each parameter 


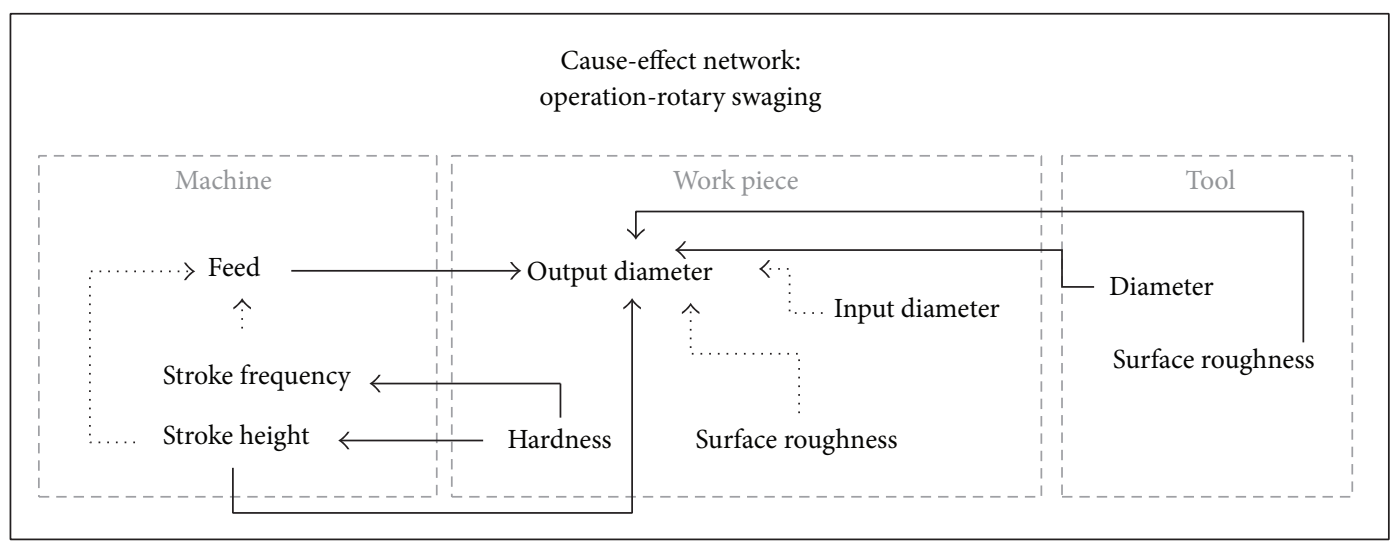

$\longrightarrow$ Cause-effect relation between material-flow elements
$\ldots .>$ Cause-effect relation within a material-flow element
$\square \downarrow$ Cause-effect network

FIGURE 4: Simplified cause-effect network for the operation "Rotary Swaging."

is assigned an interval of valid values. For example, some machines can only use tools with a specific range of sizes or operate using specific feed rates.

3.2. Simultaneous Engineering Procedure Model. The $\mu$ ProPlAn procedure model aims to support the development of new process chains in the context of a running production. Thereby, it relies on the existence of premodelled materialflow elements, although it is easily possible to add additional machines, tools, and so forth during the development. The procedure model utilizes concepts of "Simultaneous Engineering" [6]. In general, "Simultaneous Engineering" describes an approach, in which the different phases of new product development, from the first basic idea to the moment when the new product finally goes into production, are carried out in parallel [14]. By parallelizing the development of the product and of the manufacturing processes, all characteristics of the product's life cycle can be taken into account early. Ignoring such issues may lead to high costs in later stages of the product development [15]. Due to the high specialization of micromanufacturing processes, available manufacturing and handling technologies might not be suitable for a provided task. Accordingly, new technologies or tools have to be developed or the product design has to be adapted. The application of Simultaneous Engineering techniques enables an early detection of such problems, which would usually emerge at later stages of the production planning [16].

The procedure model consists of three phases (Figure 5). During the first phase, the overall process chain is developed and configured. Thereby, the process designer includes required manufacturing, handling, and quality inspection processes and defines the processes' technological interfaces, based on the product structure. In case of changes to the product structure, this phase can be repeated to adapt the model. The second phase covers the specification and configuration of the required resources. Thereby, the process designer selects suitable technologies and machines for each operation and combines their respective cause-effect networks. The last phase covers the model analysis. As a first step, he evaluates the cause-effect networks to ensure the technical feasibility of the process chain concerning the product's and the respective machines' tolerances. In a second step, valid process chains can be analyzed by means of a material-flow simulation, in order to determine and compare their logistic performances, for example, throughput times, due date adherence, workloads, or total costs. Thereby, it is possible to compare different process chains, for example, by varying used technologies, machines, or configurations, in order to determine the optimal setup and configuration.

3.3. Model Evaluation. The evaluation of the modelled production system is split in two parts: first, the evaluation of a process chain's configuration, and thus, its technological feasibility and second, the evaluation of its logistic performance. In order to validate the technological feasibility, all parameter values are propagated throughout the complete process chain using the quantitative cause-effect relations. Thereby, each parameter is calculated based on its input parameters and checked against the specified tolerances with respect to the technological interfaces and to the machines capabilities. If a parameter exceeds these tolerances, the configuration has to be adapted or, in the worst case, a more suitable technology or machine has to be selected.

After defining one or more technically feasible process chains, these models can be evaluated with reference to their logistic performance. Therefore, the process chain model is converted into a material-flow simulation, whereby the cause-effect networks' parameters are directly conveyed into the simulation. Due to the integration of technological and logistic parameters within the cause-effect networks, changes to technological parameters can directly influence their logistic counterpart. For example, an increasing feed rate usually decreases the duration, while increasing the rejection 
TABLE 1: Regression and learning methods included in the $\mu$-ProPlAn software prototype.

\begin{tabular}{|c|c|}
\hline Method & Description \\
\hline Linear regression & $\begin{array}{l}\text { As fundamental multivariate regression function, } \mu \text {-ProPlAn } \\
\text { offers the option to perform a simple linear regression on the } \\
\text { provided data. As a result, a function of the form } \\
y=\sum_{i=1}^{n}\left(a_{i} \cdot x_{i}\right)+b \text { is determined. Thereby, the least squares } \\
\text { method is applied to minimize the distance between the } \\
\text { regression model and the original data points. Additionally, } \\
\mu \text {-ProPlAn offers additional functionality to linearize, for } \\
\text { example, exponential or logarithmic data to enable linear } \\
\text { regressions. }\end{array}$ \\
\hline Polynomial regression & $\begin{array}{l}\text { In case of univariate cause-effect relations, a polynomial } \\
\text { regression can be conducted to achieve functions of the form } \\
y=a_{0}+a_{1} x+a_{2} x^{2}+a_{3} x^{3}+\cdots+a_{n} x^{n}+\varepsilon \text {. This method again } \\
\text { uses the least squares method. }\end{array}$ \\
\hline Tree-/rule-based regressions (e.g., [8]) & $\begin{array}{l}\text { In contrast to the methods above, tree- or rule-based } \\
\text { approaches do not result in a single analytic function. In } \\
\text { general, they divide the search space into smaller segments, } \\
\text { for which a regression can be performed. Usually, both } \\
\text { methods use linear regressions for each segment. }\end{array}$ \\
\hline Locally weighted linear regression (LWL) (e.g., [9]) & $\begin{array}{l}\text { LWL constitutes an abstract prediction model, which } \\
\text { performs a locally weighted linear regression each time a } \\
\text { prediction is requested. Thereby, a kernel function is used to } \\
\text { weight adjacent data points and finally a linear regression is } \\
\text { performed. This method particularly excels in interpolating } \\
\text { missing data in between available data points. }\end{array}$ \\
\hline Support vector regression(e.g., [10]) & $\begin{array}{l}\text { A support vector machine is usually used as classifier. } \\
\text { Thereby, it learns a model, which separates a set of data } \\
\text { points in one or more classes, maximizing the distance } \\
\text { between each data point and the classification curve. The } \\
\text { same method can be used for regression, particularly if the } \\
\text { provided data contains strong variances. }\end{array}$ \\
\hline Neuronal networks & $\begin{array}{l}\text { A neural network usually consists of a number of layers, each } \\
\text { containing a defined number of nodes. The nodes of each } \\
\text { layer are interconnected. During the supervised training of } \\
\text { the network, these connections' weights are adapted to } \\
\text { recreate the desired output on the last layer. Thereby, the } \\
\text { quality of the prediction strongly depends on a suitable } \\
\text { network structure (i.e., number of layers/nodes, selected } \\
\text { activation functions, etc.) }\end{array}$ \\
\hline
\end{tabular}

rate of an operation. The material-flow simulation provides several statistics regarding the underlying production system. General statistics refer to the throughput times, the duedate adherence, the single devices utilization, the work-inprogress, and the costs incurred. For each machine, more specific statistics regarding costs, down times, rejection rates, and so forth are recorded to enable a comprehensive evaluation of a single process chain as well as a comparison between different process chain models.

\section{Use-Case Scenario}

This section describes the application of $\mu$-ProPlAn for the configuration of a microrotary swaging process. Although $\mu$ ProPlAn was originally designed to adjust several processes along a process chain, the same methods can be applied for the optimization of a single process within a given process chain.
4.1. Rotary Swaging Process. Rotary swaging is a well-known cold forming process, for example, in the automotive industry. Specific characteristics of this process support lightweight and stress optimized designs of tubular components like steering columns or drive shafts [17]. The workpiece forming takes place in the swaging head in incremental steps by radial oscillating dies. Two process types are established: infeed swaging and plunge swaging that differs in the direction of the feed motion (Figure 6(a)).

An ongoing demand for miniaturization and functional integration of mechatronic systems, for example, in medical applications (minimally invasive diagnosis and surgery) or automotive industry (miniature pumps and valves), increasingly brings rotary swaging into the focus of interest, particularly on the microscale. Since 2007 , research is performed to adapt the rotary swaging process to microcomponents, with a diameter between 0.3 and $1.0 \mathrm{~mm}$ (c.f. Figure 6(b)) $[18,19]$. Actually, the prediction of the resulting workpiece's 


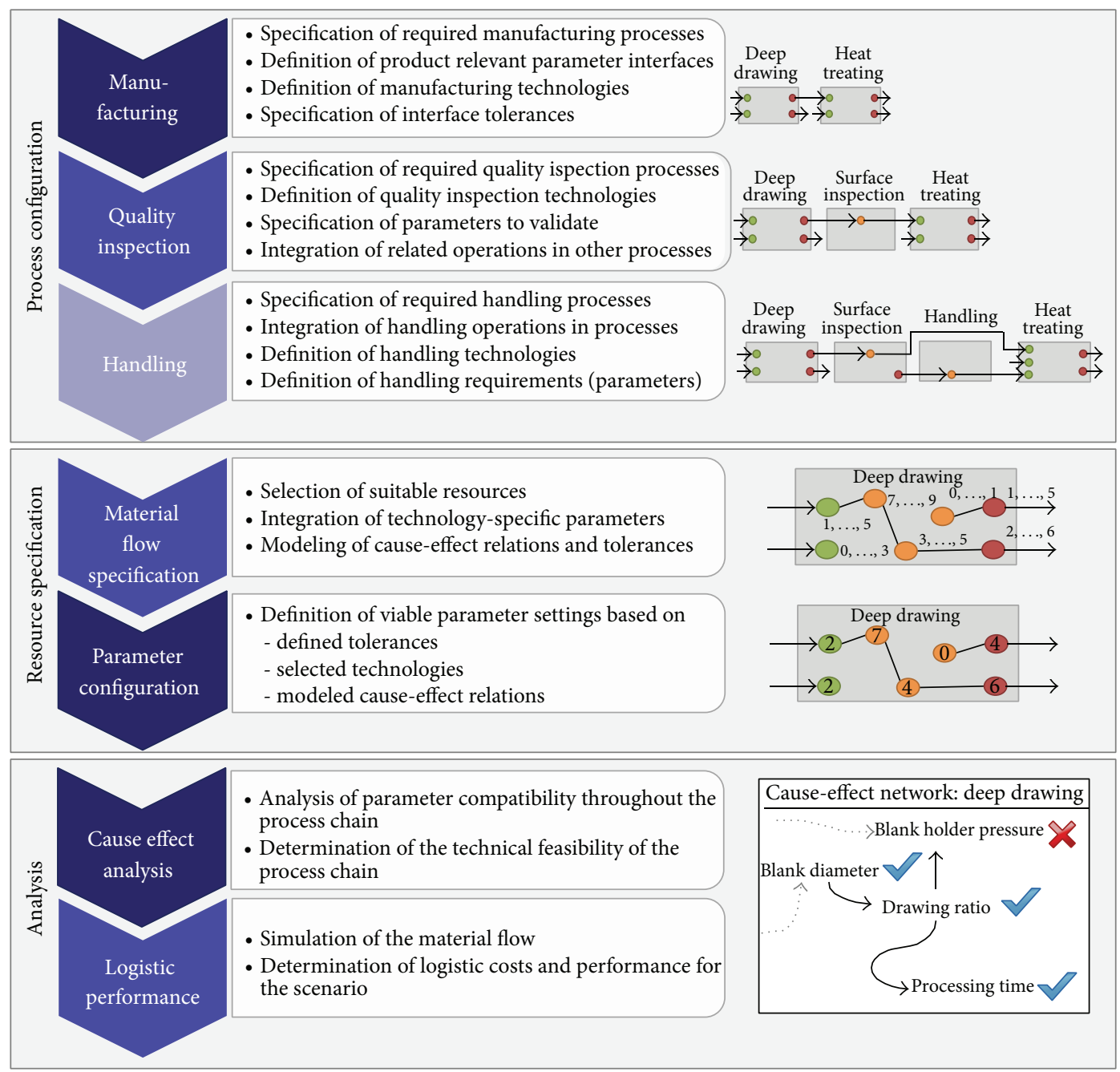

Figure 5: $\mu$-ProPlAn procedure model.

quality, for example, the final diameter with respect to the process parameters, is insufficient, even in the macroscale [20]. Material properties (e.g., its hardness), the die closing pressure, the dies' oscillation frequency, and the feed rate mainly affect the remaining spring-back, which leads to a difference between the closed dies' diameter and the finished workpiece's diameter.

4.2. Model Definition and Simulation Study. In general, the process chain for this use-case scenario consists of four processes. First, the raw material, a steel wire with a diameter of $1 \mathrm{~mm}$, is heat treated to increase its degree of deformation and to achieve a consistent hardness. Then, the described rotary swaging process is applied to decrease the diameter. Afterwards, a laser-based free from heating process is applied to create a mass accumulation at the end of the wire. Finally, plunge rotary swaging or another cold forming process is used to calibrate the mass accumulation into its final shape. As the primary focus of the experiment was the determination of the most cost-efficient setup in manufacturing steel wires with a defined diameter and mechanical properties (second process), the remaining processes were considered constant within the model. Thus, they were assigned a fixed duration and a fixed rejection rate independent of the microrotary swaging process' configuration.

In order to determine the most cost-efficient configuration for the microrotary swaging process, a set of 55 experiments was conducted using the described device. For these experiments, each of the three primary machining parameters (feed rate, oscillation frequency/stroke frequency, and die closing pressure) was modified, while the other two were held constant. As a result, the diameter of the resulting wire was measured seven times along the wire, with a distance of $1 \mathrm{~cm}$ between each measurement. Each experiment was repeated four times to determine process variances. For all experiments, the remaining process parameters were held constant, while the same material and tools were used to reduce their effects on the process and to simplify the causeeffect network. In case of the experimental setup, the die closing pressure is regulated using an additional, adjustable wedge, inflicting pressure on the dies (compare Figure 6(a)). 
TABle 2: Parameter definition.

\begin{tabular}{|c|c|c|c|c|}
\hline Parameter & & Unit & Quantitative function & Valid values \\
\hline Die closing pressure & $P$ & [Position] & - & {$[69 ; 73]$} \\
\hline Oscillation frequency & $F$ & {$[\mathrm{~Hz}]$} & - & {$[30 ; 120]$} \\
\hline Feed rate & $V_{\mathrm{f}}$ & {$[\mu \mathrm{m} / \mathrm{s}]$} & - & {$[1000 ; 4000]$} \\
\hline Initial diameter & $D_{0}$ & {$[\mu \mathrm{m}]$} & - & {$[350 ; 1000]$} \\
\hline Final diameter & $D_{1}$ & {$[\mu \mathrm{m}]$} & $D_{1}=D_{T}-35.20195 \cdot P+9.747 \cdot \ln \left(V_{f}\right)-0.36423 \cdot F+2.472 e 03$ & {$[300 ; 1000]$} \\
\hline Final diameter variance & $D_{\mathrm{v}}$ & {$[\mu \mathrm{m}]$} & Random value in between 0 and 2.5 & {$[0 ; 1000]$} \\
\hline Tool-diameter & $D_{\mathrm{T}}$ & {$[\mu \mathrm{m}]$} & - & {$[300 ; 1000]$} \\
\hline Output-length & $L$ & {$[\mu \mathrm{m}]$} & - & {$[1000 ; \infty]$} \\
\hline Tolerance & $T$ & {$[\%]$} & - & {$[0 ; 100]$} \\
\hline Operation-duration & $\mathrm{O}_{\mathrm{T}}$ & [Time Units] & $O_{T}=L / V_{\mathrm{f}}$ & {$[0 ; \infty]$} \\
\hline Operation-rejection rate & $O_{\mathrm{R}}$ & [\%] & $O_{R}=\max \left(2,\left(100 / D_{1}\right) \cdot\left(D_{v}-T\right)\right)$ & {$[2 ; 100]$} \\
\hline
\end{tabular}

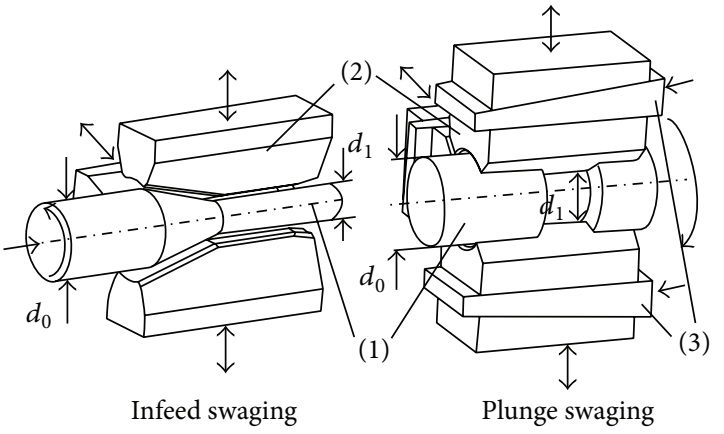

(1) Work piece

(2) Die

(3) Wedge

(a) Principle of rotary swaging

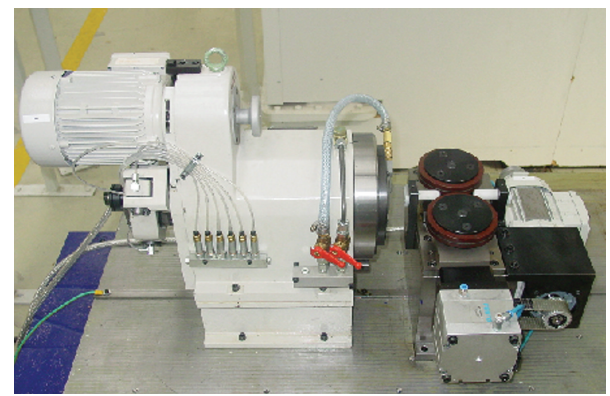

(b) Rotary swaging machine

FIGURE 6: Infeed rotary swaging.

The material-flow elements (workpieces, the rotary swaging device, and the tools) were created and assigned all relevant parameters. For the rotary swaging operation, a cause-effect network was constructed as sketched in Figure 7. Thereby, the final diameter is assumed to depend on the tool diameter as well as on the three machining parameters. For the rotary swaging operation, the duration depends on the feed rate and the desired length of the final wire, while its rejection rate depends on a specified tolerance and the maximal variance of the final diameter. The variance itself is assumed to depend on the machining parameters.
In order to quantify the cause-effect network, the data was first preexamined. In a first step, the single relations between the machining parameters and the final diameter were investigated, to enable a suitable selection for the learning/regression algorithm. Therefore, a small subset of experiments was taken from the training data, in which only one of the respective parameters varied. As depicted in Figures 8(a)-8(c) the machining parameters showed a linear, respectively, logarithmic influence. Consequently, the final diameter was quantified using a generalized adaptive linear regression model of the form $D_{1}=D_{T}+c_{1} \cdot P+$ $c_{2} \cdot \ln \left(V_{f}\right)+c_{3} \cdot F+c_{4}$. As a result of the regression, the coefficients were determined as provided in Table 2. Thereby, a residual standard error of 3.287 remained while the multiple $R$-squared value of 0.992 (adjusted $R$-squared: 0.9915$)$ was achieved.

Another result of this preliminary investigation showed a clear process window for each of these parameters (summarized in Table 2). Exceeding these windows generally leads either to strong, visible deformations of the final workpiece or to insufficient deformation results. In a second step, the diameters variance was characterized as the standard deviation over the distinct measurements of the final diameter for each experiment. Unfortunately, no covariance between the variance and any other parameter within the cause-effect network could be determined. Due to the consistent variance between 0 and $2.5 \mu \mathrm{m}$ for all final diameters between $370 \mu \mathrm{m}$ and $570 \mu \mathrm{m}$ (Figure $8(\mathrm{~d})$ ), the variance was treated as a random variable. Nevertheless, some experiments resulted in higher variances, assumingly due to material defects. In order to include these outliers in the model, a minimum rejection rate of $2 \%$ was assumed. Table 2 summarizes the parameters together with their valid ranges and quantitative functions.

4.3. Simulation Model and Results. In general, for a given set of material-flow elements (machine, tool, workers, etc.), the balancing of the process duration and its rejection rate (based on the quality of the resulting workpieces) implicates a major task in the process configuration, in order to balance the incurring process and material costs. Nevertheless, the quantification and the respective preevaluation of the data on the one hand demonstrated that the process behaved very 

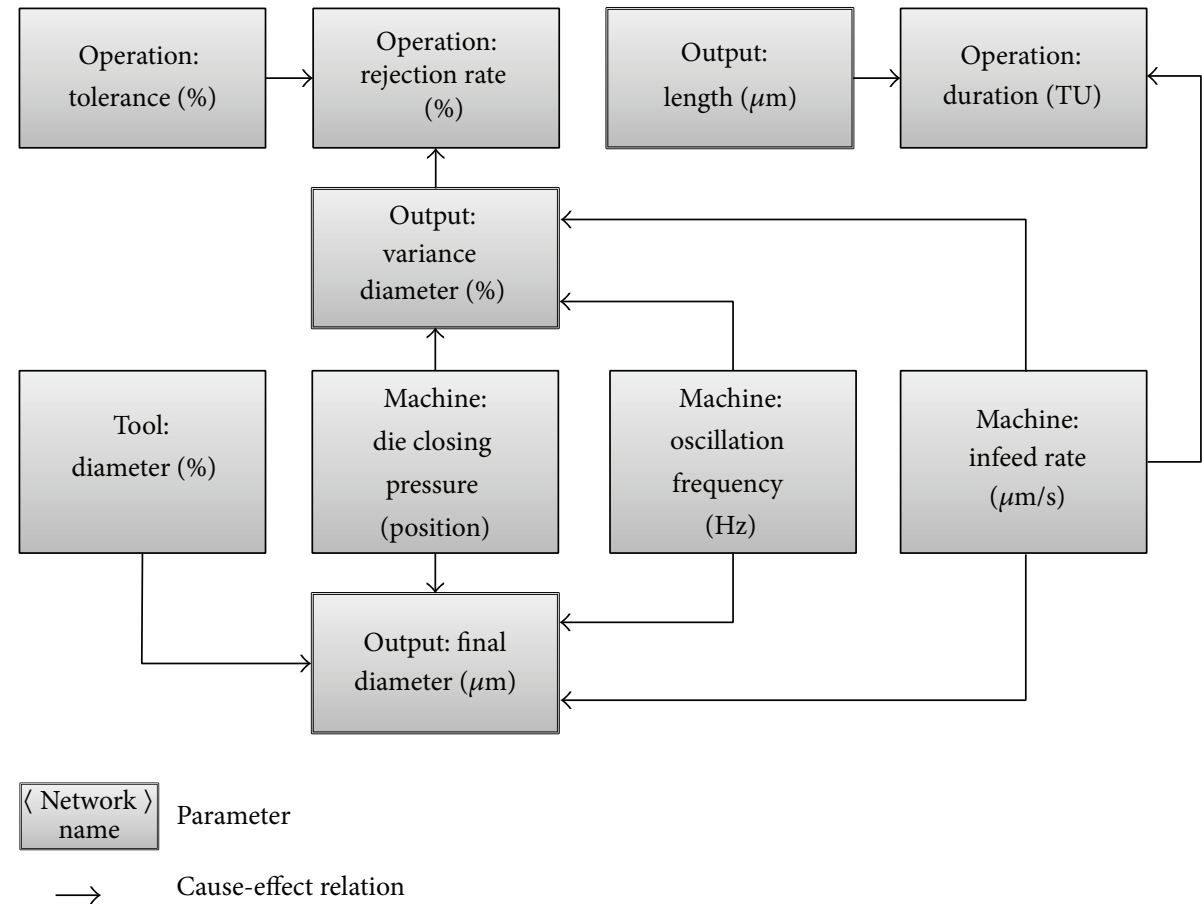

FIGURE 7: Cause-effect network for the rotary swaging operation.

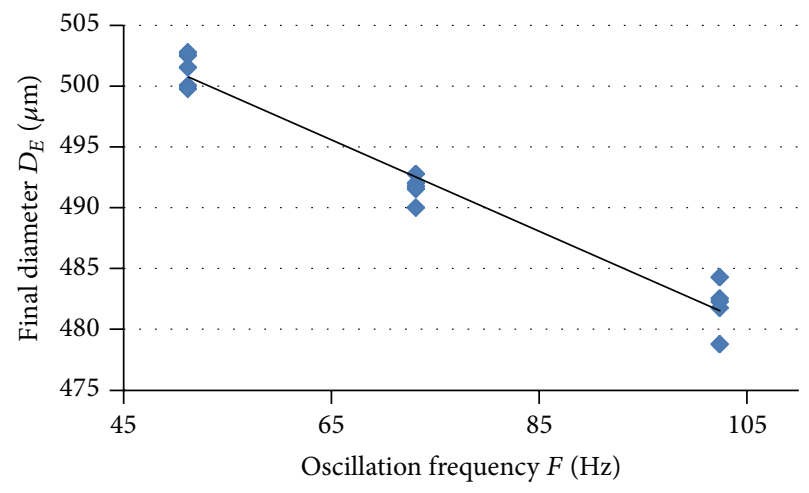

(a)

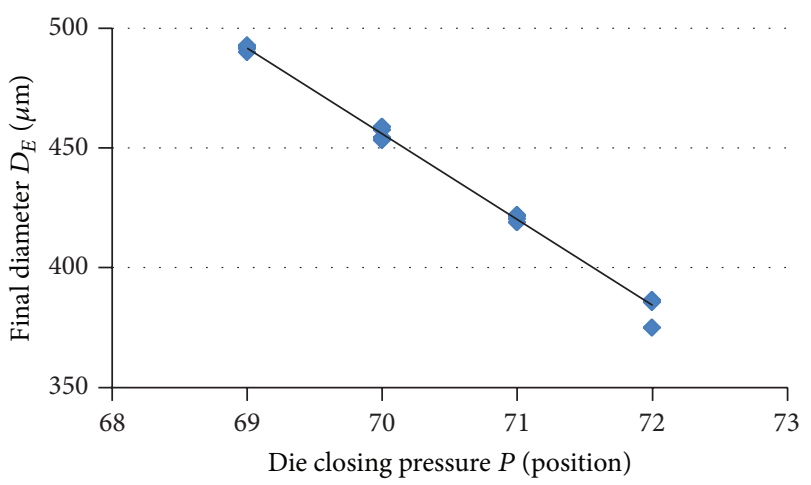

(c)

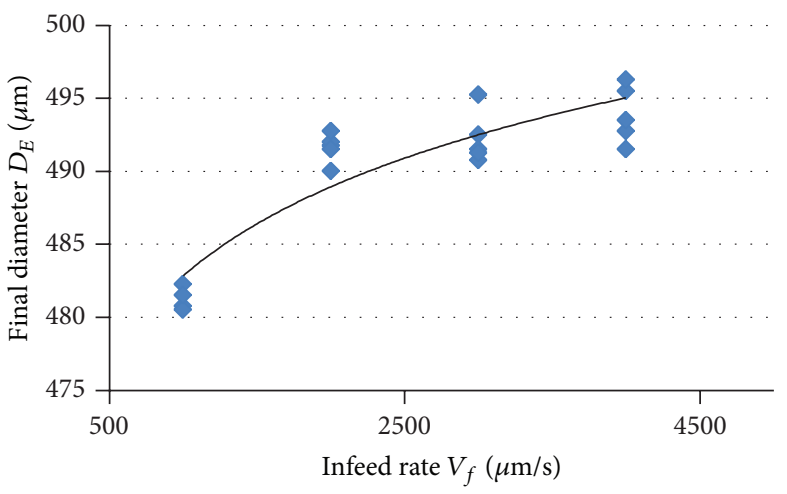

(b)

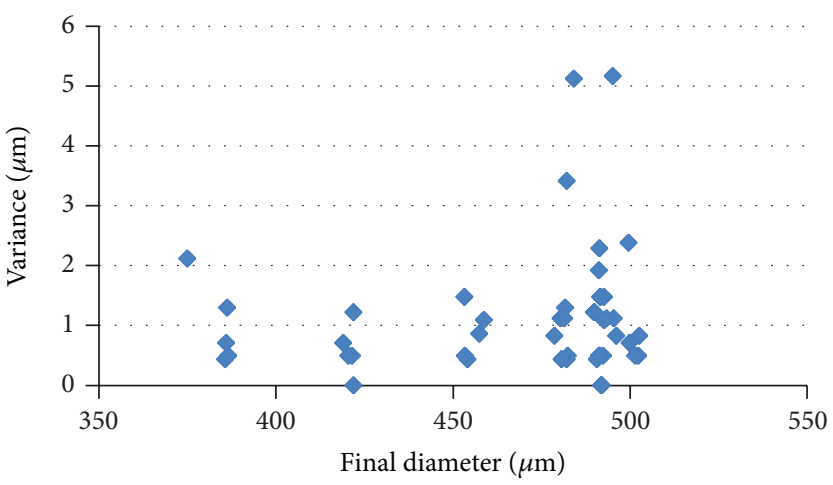

(d)

FIGURE 8: Scatterplots of selected experiments relating to the influence of single machining parameters (a)-(c) and to the distribution of the final diameters variance (d). 
TABLE 3: Parameter configurations used in the second simulation scenario.

\begin{tabular}{|c|c|c|c|c|c|}
\hline $\begin{array}{l}\text { Final } \\
\text { diameter } \\
{[\mu \mathrm{m}]}\end{array}$ & $\begin{array}{c}\text { Tool } \\
\text { diameter } \\
{[\mu \mathrm{m}]}\end{array}$ & $\begin{array}{c}\text { Die closing } \\
\text { pressure } \\
\text { [position] }\end{array}$ & $\begin{array}{c}\text { Feed rate } \\
{[\mu \mathrm{m} / \mathrm{s}]}\end{array}$ & $\begin{array}{c}\text { Oscillation } \\
\text { frequency } \\
{[\mathrm{Hz}]}\end{array}$ & $\begin{array}{c}\text { Process } \\
\text { duration } \\
{[\mathrm{sec}]}\end{array}$ \\
\hline 300 & 300 & 72.0 & 3000 & 36,587 & 33 \\
\hline 350 & 300 & 70.0 & 2000 & 87,808 & 50 \\
\hline 400 & 300 & 69.0 & 1350 & 36,587 & 74 \\
\hline 350 & 400 & 73.0 & 2100 & 73,173 & 48 \\
\hline 400 & 400 & 71.5 & 2000 & 80,491 & 50 \\
\hline 450 & 400 & 70.0 & 2000 & 87,808 & 50 \\
\hline 500 & 400 & 69.0 & 2000 & 43,904 & 50 \\
\hline 450 & 500 & 73.0 & 2100 & 73,173 & 48 \\
\hline 500 & 500 & 71.5 & 2050 & 80,491 & 49 \\
\hline 550 & 500 & 70.0 & 2000 & 80,491 & 50 \\
\hline 600 & 500 & 69.0 & 1350 & 36,587 & 74 \\
\hline
\end{tabular}

TABLE 4: Simulation results.

\begin{tabular}{lcc}
\hline & Scenario 1 & Scenario 2 \\
\hline Simulation statistics & & 50358.8 \\
Simulated time elapsed (time units) & 76838.0 & 1000.0 \\
Number of jobs finished & 1000.0 & 2974,1 \\
WIP & 5048,0 & 1492,5153 \\
Machine statistics & & 1568,2296 \\
Average queue length (Jobs) & 2581,7653 & 100,0000 \\
Maximum queue length (Jobs) & 3104,7659 & 50358,8000 \\
Average utilization (incl. setup) (\%) & 100,0000 & 49938,8000 \\
Average utilization (incl. setup) (time units) & 76838,0000 & 99,1660 \\
Average utilization (excl. setup) (time units) & 50000,0000 & 65,0720 \\
Average utilization (excl. setup) (\%) & & \\
\hline
\end{tabular}

stable, as long as all parameters remained within their respective windows. In contrast to other processes investigated using $\mu$-ProPlAn (e.g., [21]), an increasing feed rate does not lead to a significant decrease of the final workpieces quality. On the other hand, the experiments showed a very strong influence of the machining parameters on the final workpiece diameter. Thereby, a broad range of final diameters could be achieved using the same tool (in terms of the die opening diameter) while altering the parameters. Actually, manufacturing takes place using a tool with a diameter of about $\pm 50 \mu \mathrm{m}$ difference to the final diameter, while producing several test components to calibrate the process parameters. Using the regression function provided in Table 2, a more precise adjustment of the parameters could be achieved. Moreover, by exploiting the wide range of final diameters achievable with a single tool, setup times can be reduced strongly. Therefore, this subsection describes a simulation study conducted to compare the classical approach with an approach based on the application of the regression model with the aim to reduce setup times.

The simulation model covers the process chain described earlier, whereby the other three processes retain a constant duration and rejection rate. The infeed rotary swaging process assumes the same machine used in the experiments in order to derive real-world information about setup times and process behavior. For this device, it is possible to change the machining parameters in-process. Once modified, the machine directly operates using the new settings. The exchange of a tool requires about 7 minutes including the de- and reconstruction of the outer casings. To ease the simulation, all wires (jobs) are presumed to have a predetermined, consistent length of $10 \mathrm{~cm}(100.000 \mu \mathrm{m})$, while the diameter varies between $300 \mu \mathrm{m}$ and $600 \mu \mathrm{m}$ in steps of $50 \mu \mathrm{m}$ depending on the job. Setup-thus a change of parameters and/or the exchange of a tool-is only performed after a job is completed. The simulation uses a dynamic job source for each type of job (diameter). Consequently, jobs are generated in a random sequence and amount for all available diameters. Thereby, the random generators use a fixed seed to ensure the same random sequence of orders for all simulation scenarios. In general, the simulation assumes a buffered production and applies priority rules for shop floor control. Priority rules enable each machine to select the most appropriate job from their buffers. For this simulation, the priority rule first selects jobs with a minimum setup time. If several jobs require the same setup time, the most urgent jobs are selected and executed. 
For the classic simulation scenario, an individual tool is used for each type of job. Thus, to manufacture a wire with a diameter of $300 \mu \mathrm{m}$ a tool with a diameter of $300 \mu \mathrm{m}$ is used, resulting in 7 tools in total. Thereby, the simulation assumes that the parameter settings for each configuration are known and no further experiments are required to restart manufacturing after the setup. For the simulation scenario that uses the parameter settings from the regression model, only three different tools are required to cover the complete range of products. Table 3 summarizes the parameter settings and tool selections used in this simulation scenario.

Each simulation scenario was conducted ten times, whereas the simulation covered the fixed production of 1000 workpieces. The approach relying on parameter reconfiguration was thereby capable of reducing the required setup time by $34 \%$. Table 4 summarizes the main results of both simulation scenarios.

\section{Conclusion and Future Work}

The application of regression models provides a suitable approach to describing the interrelations between different parameters in a manufacturing context. Particularly for domains such as micromanufacturing, in which the specific effects of parameter changes are unknown or barely describable, such models can provide a deeper understanding of the processes based on experimental data. As demonstrated, a more detailed understanding of these effects can be used in the development of process control strategies, for example, to avoid expensive setup operations or to reduce process times in general. Future work in this use-case will deal with the inclusion of additional data and parameters to the cause-effect networks (e.g., material properties) to cover a wider range of manufacturing scenarios. In addition, more elaborate production controllers or production control strategies will be developed based on the extended cause-effect networks. In general, future work will focus on extending $\mu$ ProPlAn by methods for the automatic deduction of suitable process configurations along a process chain. This will enable a more precise adjustment of single processes. Moreover, future work will investigate possible methods to combine discrete information derived from single experiments with continuous sensor data gained during production. By combining these different types of values, more detailed causeeffect networks can be created and the acquisition of data, particularly in industrial applications, can be eased.

\section{Conflict of Interests}

The authors declare that there is no conflict of interests regarding the publication of this paper.

\section{Acknowledgment}

The authors gratefully acknowledge the financial support by the Deutsche Forschungsgemeinschaft (DFG, German Research Foundation) for Subproject C4 "Simultaneous Engineering" and Subproject A4 "Material Displacement" within the CRC 747 (Collaborative Research Center).

\section{References}

[1] J. P. Wulfsberg, T. Redlich, and P. Kohrs, "Square foot manufacturing: a new production concept for micro manufacturing," Production Engineering-Research and Development, vol. 4, no. 1, pp. 75-83, 2010.

[2] F. Vollertsen, H. S. Niehoff, and Z. Hu, "State of the art in micro forming," in Proceedings of the 1st International Conference on New Forming Technology, Harbin Institute of Technology Press, Harbin, China, 2004.

[3] Y. Qin, "Micro-forming and miniature manufacturing systems-development needs and perspectives," Journal of Materials Processing Technology, vol. 177, no. 1-3, pp. 8-18, 2006.

[4] O. Klemd, "Desktop factory-new approaches for lean micro assembly," in Proceedings of the IEEE International Symposium on Assembly and Manufacturing (ISAM '07), pp. 161-165, Ann Arbor, Mich, USA, July 2007.

[5] F. Vollertsen and R. Walther, "Energy balance in laser-based free form heading," CIRP Annals-Manufacturing Technology, vol. 57, no. 1, pp. 291-294, 2008.

[6] B. Scholz-Reiter, M. Lütjen, and N. Brenner, "Technologieinduzierte Wirkungszusammenhänge in der MikroproduktionEntwicklung eines Modellierungskonzepts," in 22. HABForschungsseminar "Digital Engineering-Herausforderung für die Arbeits- und Betriebsorganisation", M. Schenk, Ed., pp. 81-102, GITO, Magdeburg, Germany, 2009.

[7] B. Scholz-Reiter, N. Brenner, and A. Kirchheim, "Integrated micro process chains," in Advances in Production Management Systems, B. Vellespir and A. Thècle, Eds., pp. 27-32, Springer, Berlin, Germany, 2010.

[8] G. Holmes, M. Hall, and E. Frank, "Generating rule sets from model trees," in Proceedings of the 12h Australian Joint Conference on Artificial Intelligence, pp. 1-12, Springer, Berlin, Germany, 1999.

[9] E. Frank, M. Hall, and B. Pfahringer, "Locally weighted naive bayes," in Proceedings of the 19th Conference in Uncertainty in Artificial Intelligence, pp. 249-256, Morgan Kaufmann, 2003.

[10] J. Platt, "Fast training of support vector machines using sequential minimal optimization," in Advances in Kernel MethodsSupport Vector Learning, B. Schoelkopf, C. Burges, and A. Smola, Eds., MIT Press, 1998.

[11] B. Scholz-Reiter and D. Rippel, "Eine Methode zum Design von Mikroprozessketten," Industrie Management, vol. 29, no. 2, pp. 15-19, 2013.

[12] E. P. DeGarmo, J. T. Black, and R. A. Kohser, Materials and Processes in Manufacturing, John Wiley \& Sons, 9th edition, 2003.

[13] B. Denkena and H. K. Tönshoff, "Prozessauslegung und integration in die Prozesskette," in Spanen-Grundlagen, B. Denkena and H. K. Tönshoff, Eds., pp. 339-362, Springer, Berlin, Germany, 2011.

[14] R. G. Schroeder and B. B. Flynn, High Performance Manufacturing: Global Perspectives, John Wiley \& Sons, New York, NY, USA, 2002.

[15] T. T. Pullan, M. Bhasi, and G. Madhu, "Application of objectoriented framework on manufacturing domain," Journal of Manufacturing Technology Management, vol. 22, no. 7, pp. 906928, 2011.

[16] G. Reinhart and J. F. Meis, "Requirements management as a success factor for simultaneous engineering-enabling manufacturing competitiveness and economic sustainability," in 
Enabling Manufacturing Competitiveness and Economic Sustainability, H. A. ElMaraghy, Ed., pp. 221-226, Springer, Berlin, Germany, 2012.

[17] B. Kuhfuss, "Hollow drive shafts-a contribution towards weight and cost reduction in automotive construction," Automotive Technology International, no. 4, pp. 106-110, 1998.

[18] B. Kuhfuss, E. Moumi, and V. Piwek, "Micro rotary swaging: process limitations and attempts to their extension," Microsystem Technologies, vol. 14, no. 12, pp. 1995-2000, 2008.

[19] B. Kuhfuss and E. Moumi, "Influence of the feedrate on work quality in micro rotary swaging," in Proceedings of the $3 \mathrm{rd}$ International Conference on Micromanufacturing (ICOMM '08), 2008.

[20] P. Groche, D. Fritsche, E. A. Tekkaya, J. M. Allwood, G. Hirt, and R. Neugebauer, "Incremental bulk metal forming," CIRP Annals-Manufacturing Technology, vol. 56, no. 2, pp. 635-656, 2007.

[21] D. Rippel, M. Lütjen, and B. Scholz-Reiter, "A framework for the quality-oriented design of micro manufacturing process chains," Journal of Manufacturing Technology Management, vol. 25, no. 7, 2014. 


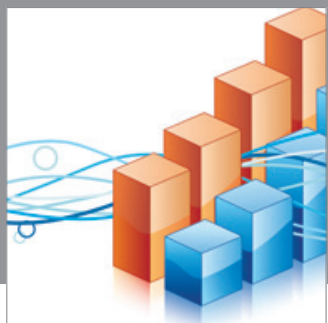

Advances in

Operations Research

mansans

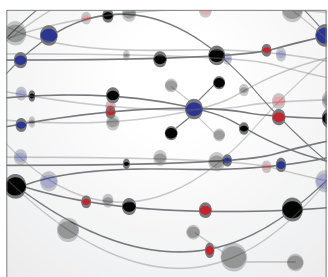

The Scientific World Journal
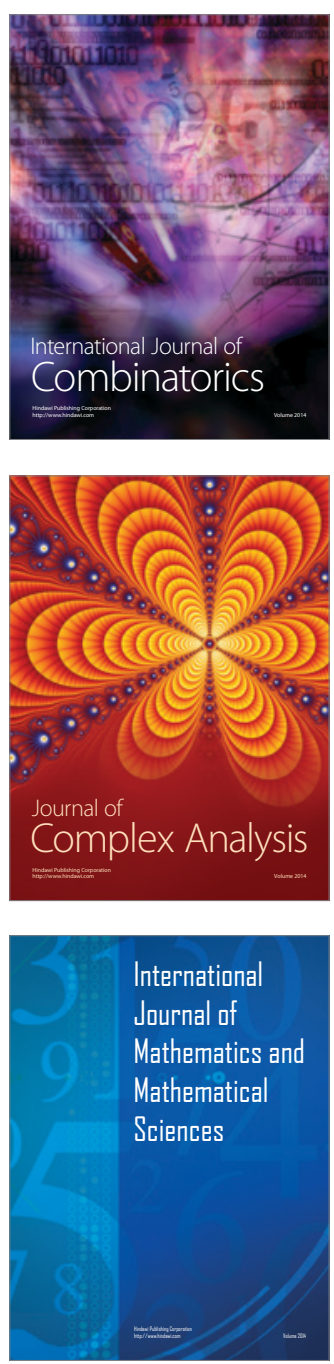
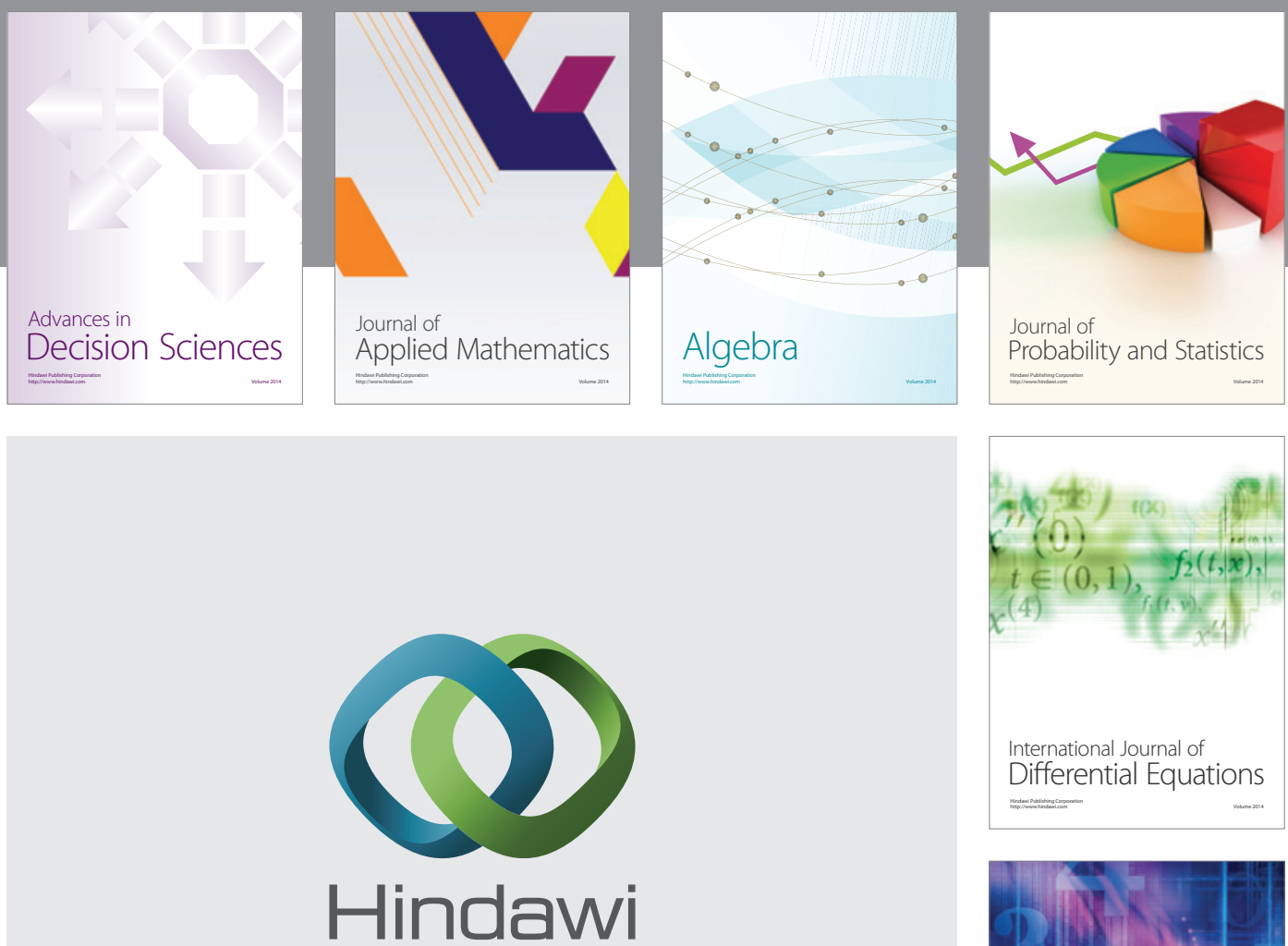

Submit your manuscripts at http://www.hindawi.com
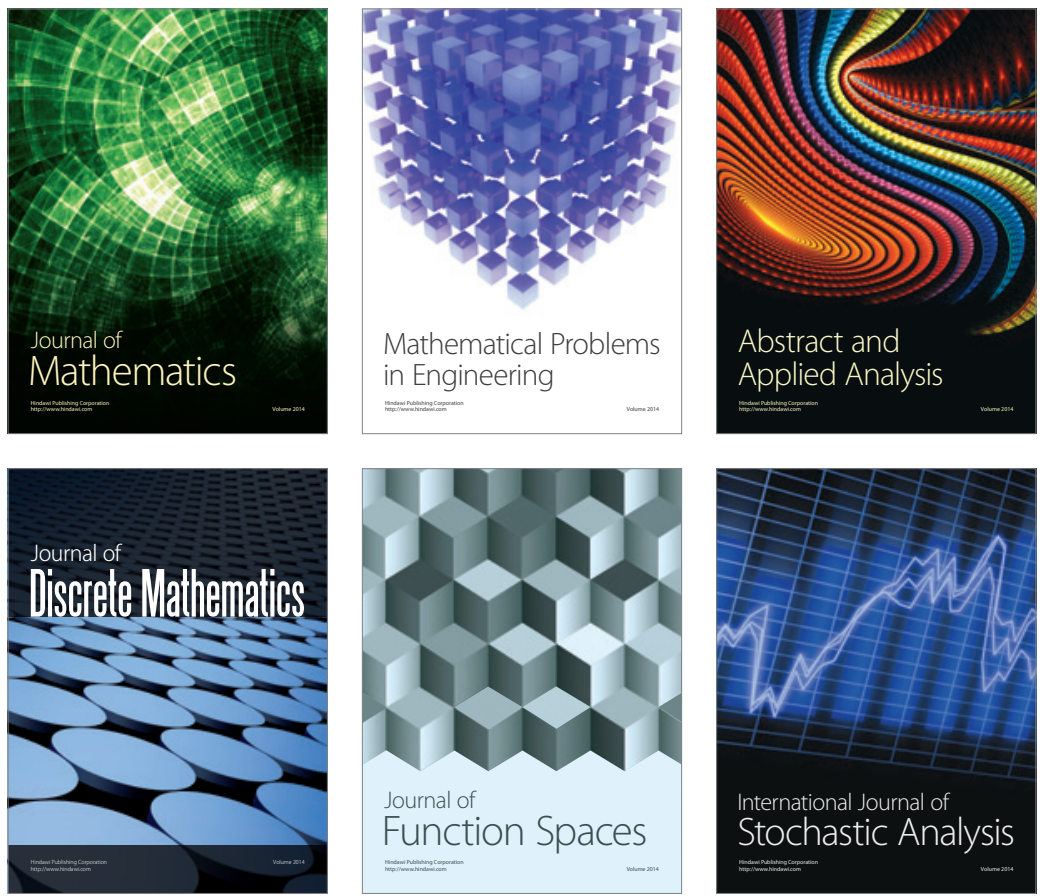

Journal of

Function Spaces

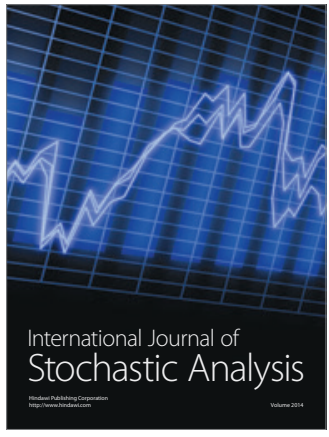

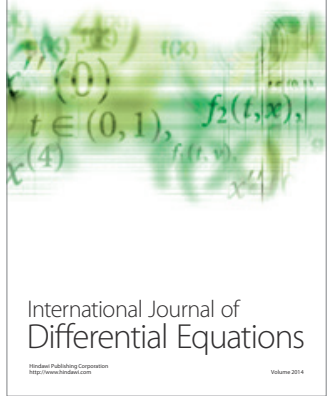
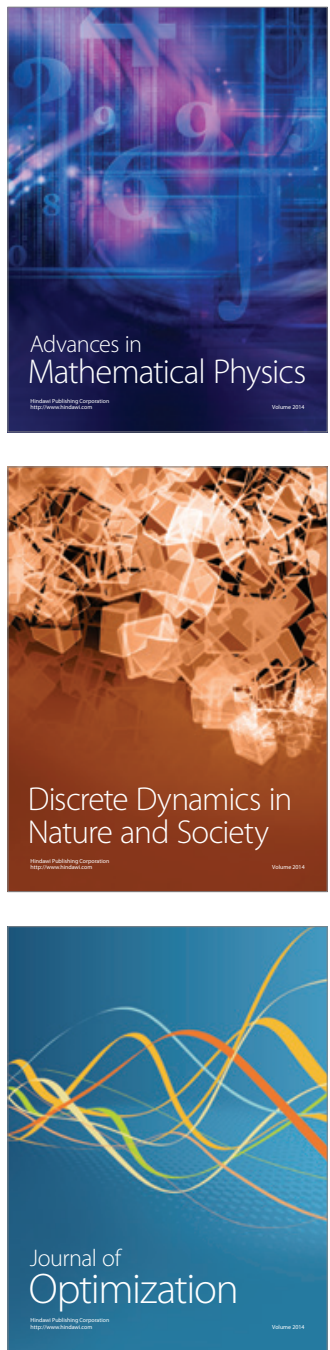